\title{
The Effect of Contralateral Acoustic Stimulation on Spontaneous Otoacoustic Emissions
}

\author{
Wei ZhaO ${ }^{1,3}$ and Sumitrajit Dhar ${ }^{1,2}$ \\ ${ }^{1}$ Roxelyn and Richard Pepper Department of Communication Sciences and Disorders, Northwestern University, 2240 Campus \\ Drive, Evanston, IL 60208, USA \\ ${ }^{2}$ The Hugh Knowles Center for Basic and Applied Hearing Research, Evanston, IL 60208, USA \\ ${ }^{3}$ Northwestern University Interdepartmental Neuroscience Program, Evanston, IL 60208, USA
}

Received: 16 June 2009; Accepted: 11 September 2009; Online publication: 2 October 2009

\begin{abstract}
Evoked otoacoustic emissions are often used to study the medial olivocochlear (MOC) efferents in humans. There has been concern that the emission-evoking stimulus may itself elicit efferent activity and alter the evoked otoacoustic emission. Spontaneous otoacoustic emissions (SOAEs) are hence advantageous as no external stimulation is necessary to record the response in the test ear. Contralateral acoustic stimulation (CAS) has been shown to suppress SOAE level and elevate SOAE frequency, but the time course of these effects is largely unknown. By utilizing the Choi-Williams distribution, here we report a gradual adaptation during the presence of CAS and an overshoot following CAS offset in both SOAE magnitude and frequency from six normal-hearing female human subjects. Furthermore, we have quantified the time constants of both magnitude and frequency shifts at the onset, presence, and offset of four levels of CAS. Most studies using contralateral elicitors do not stringently control the middle-ear muscle (MEM) reflex, leaving the results difficult to interpret. In addition to clinically available measures of the MEM reflex, we have incorporated a sensitive laboratory technique to monitor the MEM reflex in our subjects, allowing us to interpret the results with greater confidence.
\end{abstract}

Keywords: medial olivocochlear efferents, spontaneous otoacoustic emissions, cochlear physiology, middle-ear muscle reflex

Correspondence to: Wei Zhao - Roxelyn and Richard Pepper Department of Communication Sciences and Disorders - Northwestern University - 2240 Campus Drive, Evanston, IL 60208, USA. Telephone: +1-847-4671787; fax: +1-847-4912523; email: weizhao2008@u. northwestern.edu
Abbreviations: CAP - Compound action potential; CAS - Contralateral acoustic stimulation; DPOAE Distortion product otoacoustic emission; MEM Middle-ear muscle; MOC-Medial olivocochlear; OAE - Otoacoustic emission; OHC - Outer hair cell; SFOAE - Stimulus frequency otoacoustic emission; SOAE - Spontaneous otoacoustic emission; TEOAE Transient evoked otoacoustic emission

\section{INTRODUCTION}

Parallel to the well-studied auditory afferent pathways, the auditory efferent system projects in a top-down manner through the corticofugal tract and ultimately targets the cochlea via the olivocochlear bundle. The medial olivocochlear (MOC) efferents, which originate from the medial superior olivary complex, innervate outer hair cells (OHCs), modify cochlear mechanics, and alter the output of the cochlea and consequently, the auditory nerve. Among the proposed functional roles of the olivocochlear efferents are the protection against acoustic trauma and the facilitation of speech perception in noise (see Guinan 2006; Robles and Delano 2008 for recent reviews).

Clinical interest in this area is increasing as faulty efferent control of cochlear mechanics has recently been demonstrated in individuals with various disorders such as learning disabilities and auditory neuropathy (Hood et al. 2003; Garinis et al. 2008). Current methods that evaluate the efferent modulation of the cochlea often lead to great variability in the direction and magnitude of the response (Siegel and Kim 1982; Moulin et al. 1993b; Williams and Brown 1997; Sun 2008b), or are contaminated by other reflexes such as the middle-ear muscle (MEM) reflex (Whitehead et 
al. 1991; Burns et al. 1993). Hence, a complete understanding of the efferent modulation of human cochlear functions not only helps elucidate the physiological basis of this phenomenon but also enables potential development of clinical tools for the assessment of the efferent pathway and its role in various pathologies.

The effects of MOC activation on cochlear mechanics and auditory nerve activity have been extensively studied in laboratory animals (Galambos 1956; Fex 1962; Mountain 1980; Siegel and Kim 1982; Murugasu and Russell 1996). The fundamental effect of MOC activation is a reduction in basilar membrane motion (Murugasu and Russell 1996; Dolan et al. 1997) due to a decrease in the gain of the cochlear amplifier. Reduced output from the cochlea then results in a reduction in auditory nerve activity (Galambos 1956; Fex 1962; Wiederhold and Kiang 1970). The effects of these efferent-induced changes in cochlear function have also been observed in otoacoustic emissions measured in the ear canal (see Mountain 1980; Siegel and Kim 1982 for initial reports).

The effects of MOC activation have been observed on two distinct time scales - a fast effect that builds up and decays on a scale of 10-100 ms (Wiederhold and Kiang 1970; Cooper and Guinan 2003) and a slow effect on a scale of 10-100 s (Sridhar et al. 1995; Cooper and Guinan 2003). At the cellular level, the fast effect is mediated by an increase of the $\mathrm{OHC}$ conductance via calcium-activated potassium channels (Housley and Ashmore 1991; Fuchs and Murrow 1992; Kakehata et al. 1993; Erostegui et al. 1994; Evans 1996; Oliver et al. 2000; Yoshida et al. 2001) which reduces the gain of the cochlear amplifier (Dallos 1992). The slow effect, on the other hand, is hypothesized to be accompanied by both an increase of the OHC conductance and a decrease of the OHC's axial stiffness (Sridhar et al. 1995; Cooper and Guinan 2003)

The knowledge of efferent physiology and function cannot easily be transferred between species due to anatomical differences (Robles and Delano 2008) as well as different degrees of interplay with other systems such as the MEM reflex. In cats, guinea pigs, and mice, the preponderance of crossed MOC fibers (two to three times that of uncrossed MOC fibers; Robles and Delano 2008) is consistent with experimental data showing the ipsilateral MOC reflex to be two to three times stronger than the contralateral reflex (Robertson and Gummer 1985; Liberman and Brown 1986; Gifford and Guinan 1987; Brown 1989; Maison et al. 2003). In humans however, the difference between the ipsilateral and the contralateral MOC reflex is much smaller (Guinan et al. 2003; Hood et al. 2003; Lilaonitkul and Guinan 2009) and the exact ratio between crossed and uncrossed MOC fibers is unknown. The degree of interplay between the MOC and the MEM reflexes is also speciesdependent. In cats, post-onset adaptation and contralateral suppression of distortion product otoacoustic emissions (DPOAEs) were preserved when middle-ear muscles were severed, but were greatly reduced after olivocochlear bundle sectioning (Liberman et al. 1996). However in rats, the MEM reflex is the main contributor to post-onset adaptation and contralateral suppression of DPOAEs, which virtually disappear after middle-ear muscle sectioning (Relkin et al. 2005). These differences highlight the necessity for speciesspecific knowledge about the physiology of the MOC efferent system and its modulatory effect on the cochlea.

In humans, where direct and invasive measurements of either basilar membrane mechanics or auditory nerve activity are not feasible, otoacoustic emissions (OAEs) provide an indirect but noninvasive probe to evaluate the effects of the MOC efferents on cochlear mechanics. In the vast majority of experiments in human subjects, the MOC efferents are stimulated acoustically to examine changes in evoked emissions, such as transient evoked OAEs (TEOAEs), distortion product OAEs (DPOAEs), and stimulus frequency OAEs (SFOAEs) (Veuillet et al. 1991; Moulin et al. 1993a; Guinan et al. 2003). Such acoustic stimulation of the MOC efferents can be conducted in the ear in which the OAE is being monitored (ipsilateral), in the opposite ear (contralateral), or in both (bilateral). The effect on the OAE is largest with bilateral stimulation (Guinan et al. 2003; Hood et al. 2003; Lilaonitkul and Guinan 2009), but both bilateral and ipsilateral stimulation are problematic due to two-tone suppression in the monitored ear. With contralateral acoustic stimulation (CAS), the issue of contamination of the observation from two-tone suppression is avoided. However, all methods of acoustic stimulation risk contamination due to the activation of the MEM reflex. Finally, the emission-evoking stimulus could itself elicit efferent activity and interact with the otoacoustic emission (Guinan et al. 2003). In this regard, Guinan et al. (2003) argued that SFOAEs provide a better assay to evaluate the MOC pathway, as higher amounts of total energy present in stimuli used to record TEOAE and DPOAEs would be more likely to evoke MOC activity. Here, we extend this argument by examining the effects of efferent activation on spontaneous otoacoustic emissions (SOAEs), a type of OAE recorded without the use of an external stimulus.

Because broadband noise is an effective activator of both the MOC efferent activity and the MEM reflex (Norman and Thornton 1993; Maison et al. 2000; Velenovsky and Glattke 2002), the possibility of contamination from the MEM reflex is ever present and vexatious, demanding in-depth treatment. The range of temporal characteristics of the MOC and 
MEM reflexes, and the overlap between them makes it difficult to isolate their influence on OAEs (Goodman and Keefe 2006). As has been discussed above, the relative contribution of the MEM reflex to OAE shifts has been found to be species-dependent in laboratory animals. While the stapedius muscle can be sectioned in animal experiments, thereby allowing the parsing of contributions from the MEM and MOC reflexes, this luxury is not available in most experiments involving human subjects. Although it is difficult to ascertain the relative roles of the MOC and MEM reflexes in humans, an approximate estimate can be obtained from groups with specific pathologies that cause the attenuation/eradication of one or the other of these reflexes. In one such published work, significantly reduced suppression of TEOAE by CAS was demonstrated in a group of patients who had undergone vestibular nerve neurotomy (severed crossed MOC efferents) (Giraud et al. 1995). In contrast, contralateral suppression of TEOAE in another group of subjects diagnosed with Bell's palsy (abolition of stapedial reflex due to paralysis of the facial nerve) was comparable to that of a control group. This led the authors to argue that the MEM reflex plays a relatively insignificant role in human subjects. However, the tensor tympani muscle was intact and active in the subjects with Bell's palsy thereby allowing the possibility of the middle ear having influenced these results.

An acceptable approach in experiments with human subjects would be to measure the MEM reflex threshold in individual subjects. This would at least grant the experimenter the knowledge that when the MOC elicitor is higher in level than the MEM reflex threshold, the outcome was possibly "helped" by the MEM reflex. Complications taint this approach as well. First, the clinical MEM reflex thresholds vary with the sensitivity of the instrumentation and the testing procedures (Gelfand 1984). Moreover, thresholds measured using clinical instruments have been consistently found to be substantially higher than those measured using a variety of experimental techniques (Neumann et al. 1996; Feeney et al. 2003; Guinan et al. 2003; Muller-Wehlau et al. 2005; Goodman and Keefe 2006). However, most studies using contralateral noise elicitors have not controlled for the MEM reflex stringently, leaving their results difficult to interpret.

Efferent modulation of SOAEs has not been studied as extensively in the literature as other evoked OAEs. Still, activation of the MOC efferents has been shown to have a consistent effect on SOAEs. CAS with tones or broadband noise as the elicitor of MOC efferents has been shown to reduce SOAE amplitude and increase SOAE frequency (Mott et al. 1989; Harrison and Burns 1993; Smurzynski et al. 2002).
The time course of CAS-induced changes in SOAE amplitude and frequency has been quantitatively characterized at the onset of CAS (Smurzynski et al. 2002). However, the temporal behavior of SOAEs during CAS or at CAS offset has not been characterized with sufficient resolution. Harrison and Burns (1993) observed a general release from MOC-induced shifts in both SOAE amplitude and frequency over a 40-s period. Nonetheless, the coarse temporal resolution of their measurement and analysis did not allow accurate description of the time course of events.

In this article, we have attempted to fully characterize the temporal behavior of magnitude and frequency shifts of SOAEs, at the onset of, during, and at the offset of four levels of contralateral broadband noise. To achieve both high temporal and spectral resolution, we have utilized the Choi-Williams distribution, a member of the Cohen class distribution function (Cohen 1995). In the execution of our experiment, we have attempted to detect the MEM reflex threshold by monitoring the magnitude and phase of a pure tone at two frequencies while stimulating the contralateral ear with broadband noise over a range of levels.

\section{METHODS}

\section{Subjects}

Six female participants (S1-S6) between the ages of 18 and 30 years with clinically normal hearing sensitivity $(20 \mathrm{~dB}$ HL or better at octave frequencies between $250-8,000 \mathrm{~Hz}$, measured by the Interacoustics Audio Traveller AA220) in both ears and at least one SOAE, greater than $10 \mathrm{~dB}$ SPL (re $20 \mu \mathrm{Pa}$; SOAE spectra shown in Figure 1) were recruited to participate. Subjects were compensated for their participa-
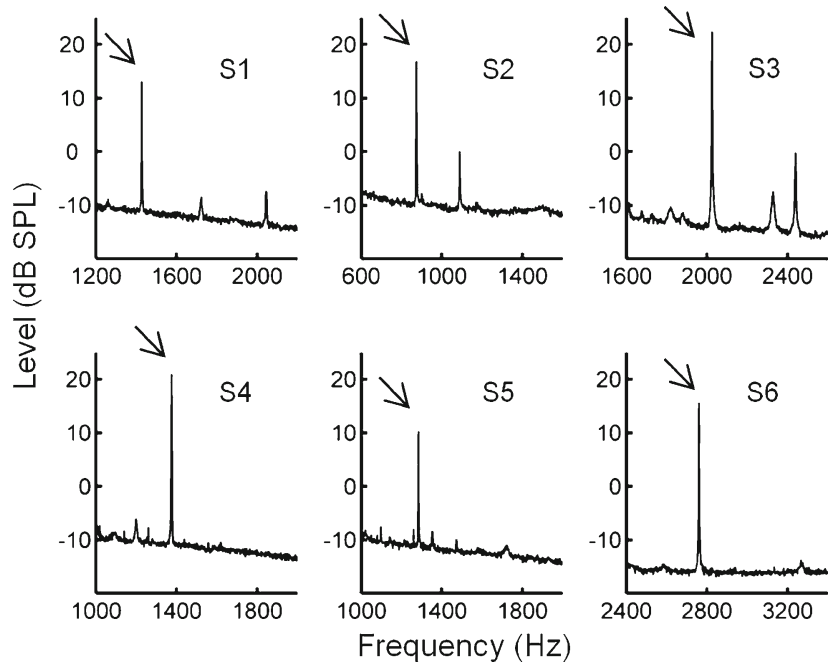

FIG. 1. SOAE spectra for all six subjects (S1-S6). The SOAEs marked by arrows were examined in detail for this study. 
tion, and the Northwestern University Institutional Review Board approved all procedures. Measurements were made in a sound-treated audiological test booth.

\section{Signal generation and recording}

Experimental stimuli were generated and digitized by a MOTU 828 MKII input/output converter controlled by a Macintosh computer via firewire. Signals were amplified by a custom-built amplifier and presented to the subjects' ears using MB Quart 13.01 HX drivers. Signals were recorded in participants' ears by an Etymotic Research ER-10B+ microphone, amplified by a preamplifier (20 dB gain), digitized by the MOTU and eventually stored on the Macintosh computer for analysis.

\section{Measuring MEM reflex}

MEM reflex thresholds were measured in each subject using the AA220 (standard probe tone at $226 \mathrm{~Hz}$, with a contralateral wideband noise as the reflex elicitor). These results along with those obtained by a novel experimental procedure (described below) are displayed in Table 1 . In addition to the measurement of MEM reflex thresholds using a clinical device, we made a set of parallel measurements by monitoring the magnitude and phase of a probe tone in the test ear while the contralateral ear was stimulated by a broadband noise. A contralateral broadband (white) noise $(100-10,000 \mathrm{~Hz}$, between 50 and $90 \mathrm{~dB}$ SPL in $10 \mathrm{~dB}$ steps) was presented as the MEM reflex elicitor. A probe tone of either 602 or $226 \mathrm{~Hz}$ at $60 \mathrm{~dB}$ SPL was monitored in the test ear using an Etymotic Research ER-10B+ microphone. In each run, the probe tone was on for $4 \mathrm{~s}$ and the contralateral elicitor was presented for $500 \mathrm{~ms}$ between 1 and $1.5 \mathrm{~s}$ (Fig. 2). A total of eight runs were recorded for each elicitor level and probe tone frequency. Averaged data for each elicitor level and probe tone frequency were passed through an adaptive least-squares fit filter (Long and Talmadge 1997) to estimate the magnitude and phase of the probe tone. The MEM reflex threshold was determined based on a time-locked change in the probe tone magnitude and phase in response to the contralateral noise. The lowest contralateral elicitor level that induced the characteristic pattern (described below) of the MEM reflex was taken as the threshold.

It was assumed that the $226 \mathrm{~Hz}$ probe tone is only sensitive to the acoustic impedance change of the middle ear induced by the MEM reflex because SFOAEs are extremely difficult to measure at this low-frequency range (Goodman and Keefe 2006). The choice of the 602-Hz probe tone was based on Feeney et al. (2003) which showed that the greatest change in reflectance due to the MEM reflex was approximately around this frequency. A positive change in the magnitude of the total pressure of the $226-\mathrm{Hz}$ probe tone, time locked to the contralateral noise was taken as an indicator of a MEM reflex. Results obtained from subject 6 are displayed in Figure 2, with results for the 226- and 602$\mathrm{Hz}$ probe tones represented in the left and right columns, respectively. Contralateral noise ranges from 50 to $90 \mathrm{~dB}$ SPL in 10-dB steps. Traces representing pressure and phase measurements are displayed for each probe tone. Note the increase in magnitude of the 226- $\mathrm{Hz}$ probe tone for a noise level of $70 \mathrm{~dB}$ SPL. A reduction in the phase of the $226-\mathrm{Hz}$ probe tone was always observed with an increase in magnitude. Thus, $70 \mathrm{~dB}$ SPL was the designated MEM reflex threshold in this subject. As demonstrated in Figure 2, the changes in the magnitude and phase of the $602-\mathrm{Hz}$ probe were more complex. A decrease in magnitude and an increase in phase were commonly observed at CAS levels below the MEM reflex threshold. Above the MEM reflex threshold, a general reduction in the phase of the 602-Hz probe was observed. However, complex patterns were often observed in both magnitude and phase at noise levels above the MEM reflex threshold, possibly indicating the interplay between the MEM reflex and the MOC reflex which alters SFOAE at the probe frequency. These observations, though interesting, are not the main focus of this paper. MEM reflex threshold estimates using the experimental method were consistently and significantly lower than those obtained using the AA220. We, therefore, chose to be guided by the MEM reflex threshold obtained by our experimental protocol.

\section{TABLE 1}

MEM reflex thresholds obtained with broadband noise in the contralateral ears of all six subjects using clinical and experimental methods

\begin{tabular}{lrrrrr}
\hline Subject \# & 1 & 2 & 3 & 4 & 5 \\
\hline Clinical MEM reflex threshold (dB SPL) & 100 & 100 & 85 & 90 & 80 \\
Experimental MEM reflex threshold (dB SPL) & 70 & 70 & 70 & 50 & 70 \\
\hline
\end{tabular}

Note the consistently lower thresholds obtained by the experimental method 


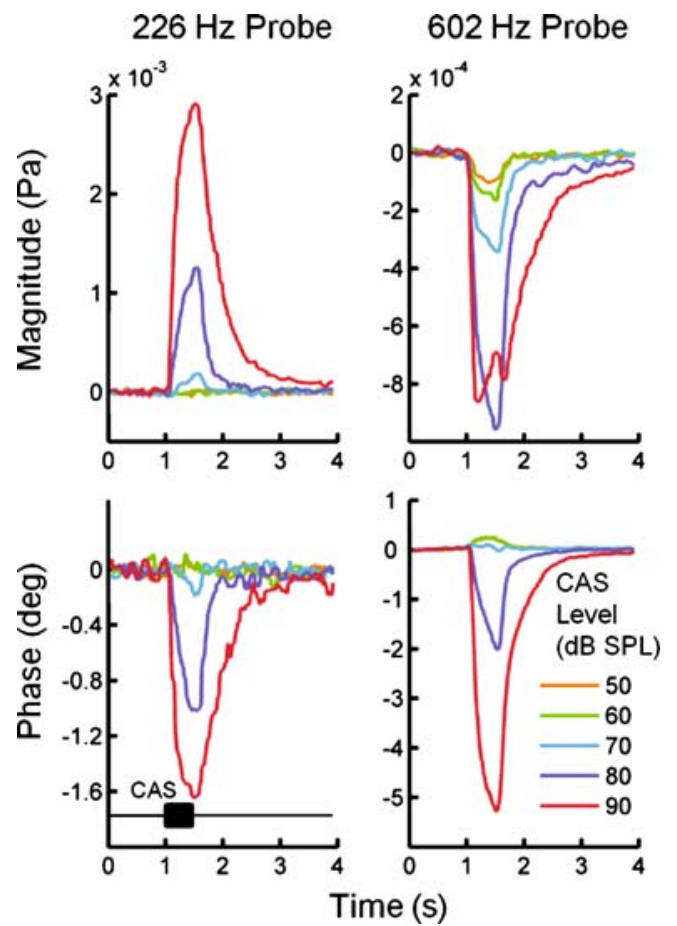

FIG. 2. Representative results of the experimental method of measuring the MEM reflex threshold (subject 6). The magnitude and phase (both normalized to baseline) of a probe tone (226 or $602 \mathrm{~Hz}$ at $60 \mathrm{~dB}$ SPL) were monitored with CAS between 50-90 dB SPL from 1 to $1.5 \mathrm{~s}$ (black bar on the bottom left). A time-locked increase in the magnitude of the total pressure at $226 \mathrm{~Hz}$ was considered a sign of the MEM reflex. The reflex threshold in this subject was determined to be $70 \mathrm{~dB}$ SPL. Interestingly, for CAS above the experimental threshold of $70 \mathrm{~dB} \mathrm{SPL}$, the phase of the $226 \mathrm{~Hz}$ probe showed a lag; the phase of the $602 \mathrm{~Hz}$ probe turned from a lead to a lag. The magnitude of the $602 \mathrm{~Hz}$ probe always showed a decrease, but it exhibited a biphasic pattern (a decrease followed by an increase) for CAS of $90 \mathrm{~dB}$ SPL.

\section{SOAE measurements}

A broadband noise $(100-10,000 \mathrm{~Hz})$ was presented in the contralateral ear while the SOAEs (without any stimulation) were monitored in the test ear. In each run, SOAEs were monitored for $35 \mathrm{~s}$ and the contralateral noise elicitor was turned on at $5 \mathrm{~s}$ and off at $25 \mathrm{~s}$ (Fig. 3). There was a 10-s break between runs. Four contralateral noise levels (45, 55, 65, $75 \mathrm{~dB}$ SPL) were interleaved and 20 runs were averaged for each noise level. Subject $4 \mathrm{had}$ a fairly low MEM reflex threshold as measured by our experimental assay, thus was not exposed to the highest level of noise at $75 \mathrm{~dB}$ SPL.

Participants' SOAEs were also measured without contralateral stimulation before and after the test.

\section{Analysis}

A time-frequency analysis using the Choi-Williams distribution function was applied in MATLAB to examine the shifts in SOAE magnitude and frequency with both fine temporal and spectral resolution. The ChoiWilliams distribution function is a time-frequency analysis tool of the Cohen's class distribution function (Cohen 1995), which renders the energy density (represented by arbitrary magnitude) of a signal $s(t)$ on a time-frequency plane, and is fundamentally different from the spectrogram. The Cohen's class distribution function has a general form as follows:

$$
\begin{gathered}
C(t, \omega)=\frac{1}{4 \pi^{2}} \iiint s^{*}\left(u-\frac{1}{2} \tau\right) s\left(u+\frac{1}{2} \tau\right) \\
\times \phi(\theta, \tau) e^{-j \theta t-j \tau \omega+j \theta u} d u d \tau d \theta
\end{gathered}
$$

where $C(t, \omega)$ is the energy density at time $t$ and frequency $\omega$. Since the energy density can be written as the Fourier transform of the deterministic local autocorrelation function $R_{\mathrm{t}}(\tau)$, we have

$$
C(t, \omega)=\frac{1}{2 \pi} \int R_{t}(\tau) e^{-j \tau \omega} d \tau
$$

By comparing Eqs. 1 and 2, we have $R_{\mathrm{t}}(\tau)$, the deterministic generalized local autocorrelation function as follows:

$R_{t}(\tau)=\frac{1}{2 \pi} \iint s^{*}\left(u-\frac{1}{2} \tau\right) s\left(u+\frac{1}{2} \tau\right) \phi(\theta, \tau) e^{j \theta(u-t)} d \theta d u$

where $\tau$ is the delay from time $u$ in the autocorrelation function and $\theta$ is an intermediate variable without a direct physical correlate. $\phi(\theta, \tau)$ is the kernel function which mitigates the inherent artifacts (crossterms) of the Cohen's class distribution function. Different kernels $\phi(\theta, \tau)$ renders different members of the Cohen's class. The Choi-Williams distribution has a kernel $\phi(\theta, \tau)$ as follows:

$$
\phi(\theta, \tau)=e^{\theta^{2} \tau^{2} / \sigma}
$$

where $\sigma$ is an adjustable parameter that is used to optimize the results. A typical example of shifts in SOAE magnitude and frequency obtained from the Choi-Williams distribution is shown in Figure 4. Specifically, we examined the SOAE shifts at the onset, in the presence, at the offset and after the offset of CAS. The shifts were fitted with a oneexponential curve (Eqs. 5 and 6).

$$
\begin{gathered}
y(t)=y_{0}+k e^{-t / \tau} \\
y(t)=y_{1}+y_{2}\left(1-e^{t / \tau}\right)
\end{gathered}
$$

where $y(t)$ is the function of magnitude or frequency change over time $t$. $y_{0}$ or $y_{1}+y_{2}$ is the asymptotic value of $y . k$ is a constant and $\tau$ is the time constant of the exponential function. 


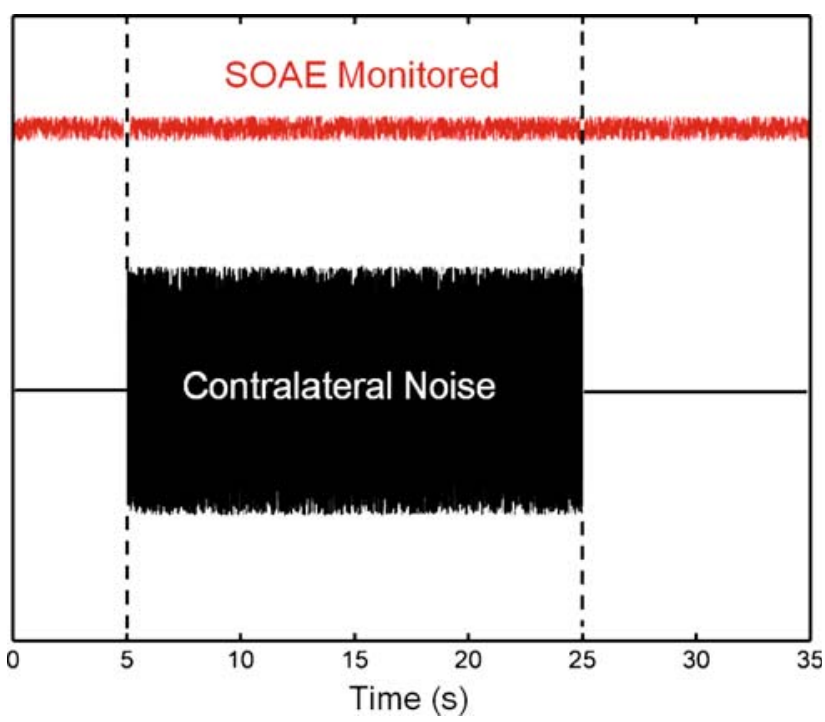

FIG. 3. An illustration of the experimental design of studying effects of CAS on SOAEs. Contralateral noise was presented between 45 and $75 \mathrm{~dB}$ SPL, in 10-dB steps during a time window between 5 and $25 \mathrm{~s}$ while the selected SOAE was monitored for $35 \mathrm{~s}$.

\section{RESULTS}

Six relatively large SOAEs (greater than $10 \mathrm{~dB}$ SPL) from six normal-hearing young healthy female human ears were examined. The examined SOAEs were between 876 and $2,764 \mathrm{~Hz}$ in frequency, and between 21 and $36 \mathrm{~dB}$ in signal-to-noise ratio (Fig. 1). As can be observed in the panels of Figure 1, each of our subjects had multiple SOAEs in the ear studied. We chose the largest SOAE for detailed examination. The level and frequency of the SOAE was monitored for a period of $35 \mathrm{~s}$, while the contralateral ear was stimulated with a broadband noise in the time window between 5 and $25 \mathrm{~s}$. The typical effect of CAS on the frequency and magnitude of an SOAE is illustrated on a timefrequency plane as in Figure 4. During CAS, the SOAE frequency was elevated (upwards shift in the plot) and magnitude reduced (change from warmer to cooler colors). We discuss the details of the CAS-induced changes in SOAE magnitude and frequency below.

\section{Effect of contralateral noise on SOAE magnitude}

The magnitude of each of the six SOAEs studied exhibited a rapid reduction at CAS onset, a gradual release from reduction (adaptation) in the presence of CAS, a rapid overshoot at CAS offset, and a gradual return to baseline (recovery) (Fig. 5). The magnitude of the SOAE was quickly reduced as contralateral noise was gated on at $5 \mathrm{~s}$. Over almost the entire 20-s CAS window, the SOAE magnitude was gradually and partially released from the reduced state. At $25 \mathrm{~s}$ when contralateral noise was gated off, the SOAE magnitude quickly recovered towards the baseline but usually overshot the baseline for a brief period of time, returning to baseline over the next few seconds. The reduction, adaptation (release from initial magnitude reduction), and the overshoot after CAS offset were more prominent in some subjects than others.

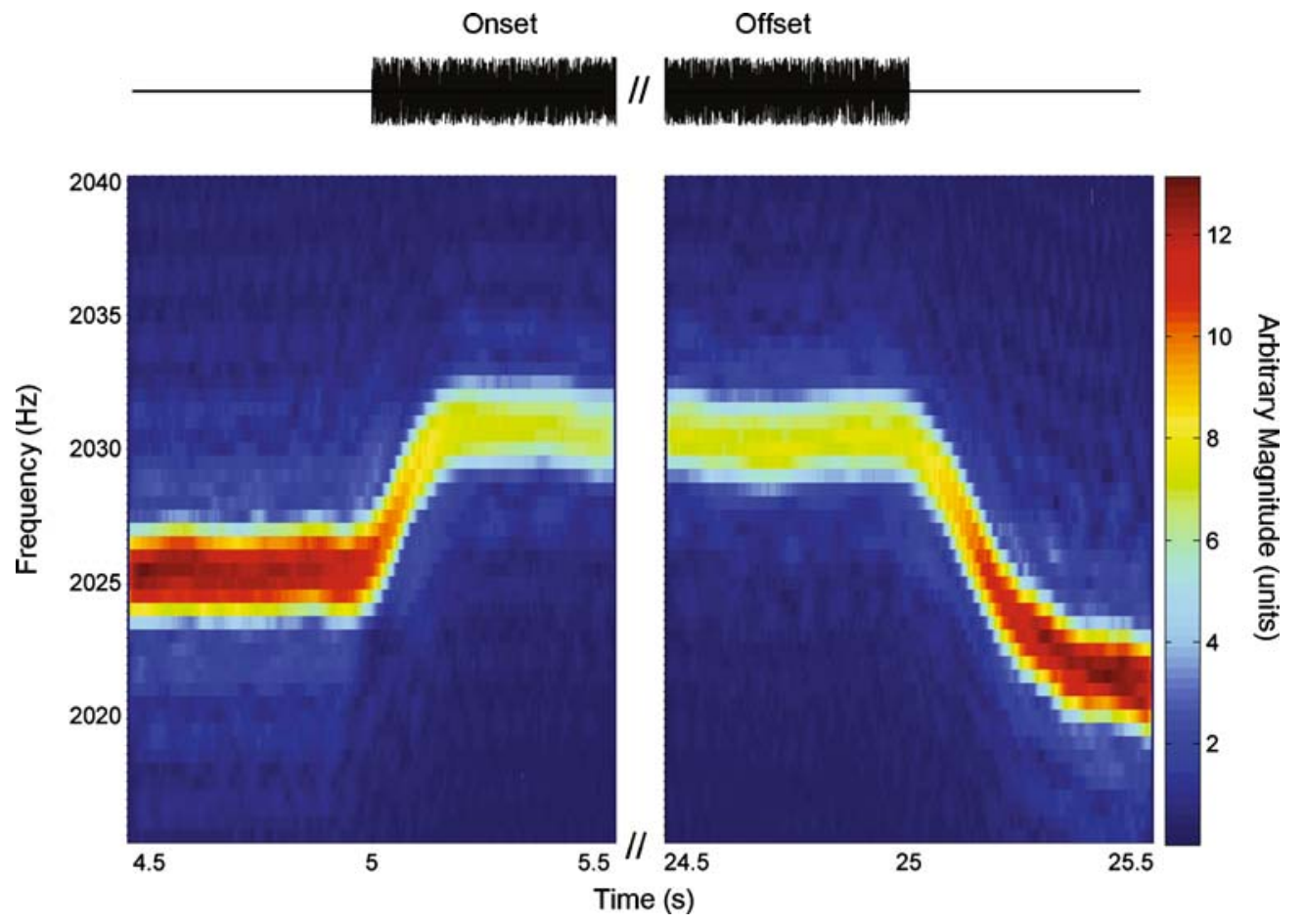

FIG. 4. A 2-D illustration of typical shifts in SOAE frequency and magnitude, obtained from the Choi-Williams distribution analysis. During CAS, the SOAE frequency was elevated (upwards shift on the plot) while magnitude was reduced (change from warmer to cooler colors). 


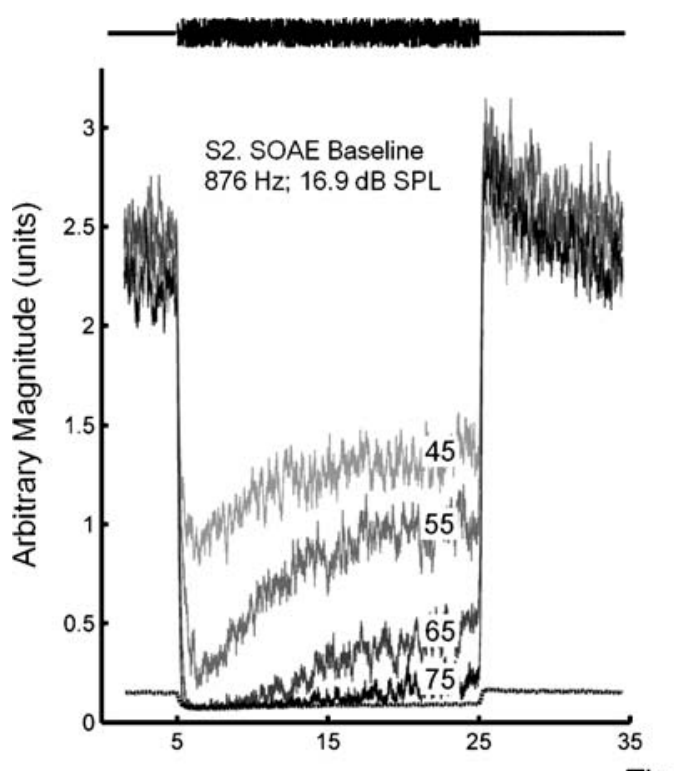

Time (s)

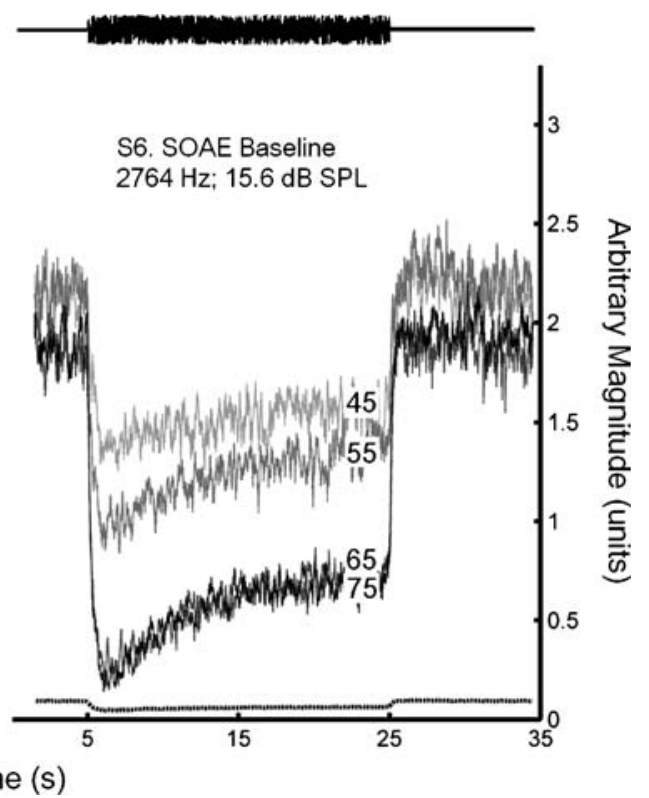

floor. The reduction of the noise floor during CAS was an artifact of the Choi-Williams analysis which relates to cross-terms. In both subjects, the MEM reflex threshold was $70 \mathrm{~dB}$ SPL, as estimated by our experimental method.

defined for comparison. Baseline was defined as the average magnitude in the 1-s window before CAS onset. The reduced state was defined as the average SOAE magnitude in a 1-s window before CAS offset. Finally, the post CAS peak was defined as the highest recorded magnitude in a 2-s time window following CAS offset. From these reference points, shift was computed as the deviation of the reduced state from the baseline. Similarly, overshoot was computed as the deviation of the post CAS peak from the baseline. A schematic illustration of these computations in subject 2 is presented in the leftmost column of Figure 6 . The top and bottom rows exhibit SOAE behaviors due to CAS of 45 and $65 \mathrm{~dB}$ SPL, respectively. Different stages of CAS-induced changes of the same SOAE are highlighted in the remaining columns of Figure 6 . Each stage was fitted with a one-exponential curve to quantify the temporal characteristics of CAS-induced initial reduction, adaptation, overshoot, and return to baseline which typically follows the overshoot. The thick red lines in each of the last four columns of Figure 6 represent these fits (see Eqs. 5 and 6 in methods). Shift, overshoot, as well as time constants for various stages depicted in Figure 6 are presented later for all subjects.

\section{Effect of contralateral noise on SOAE frequency}

Figures 7 and 8 are analogs of Figures 5 and 6, but show changes in SOAE frequency rather than magnitude. As was observed in the case of SOAE magnitude, SOAE frequency demonstrated a rapid change (ele-

In an attempt to fully characterize all stages of CAS induced changes to an SOAE, three states were 


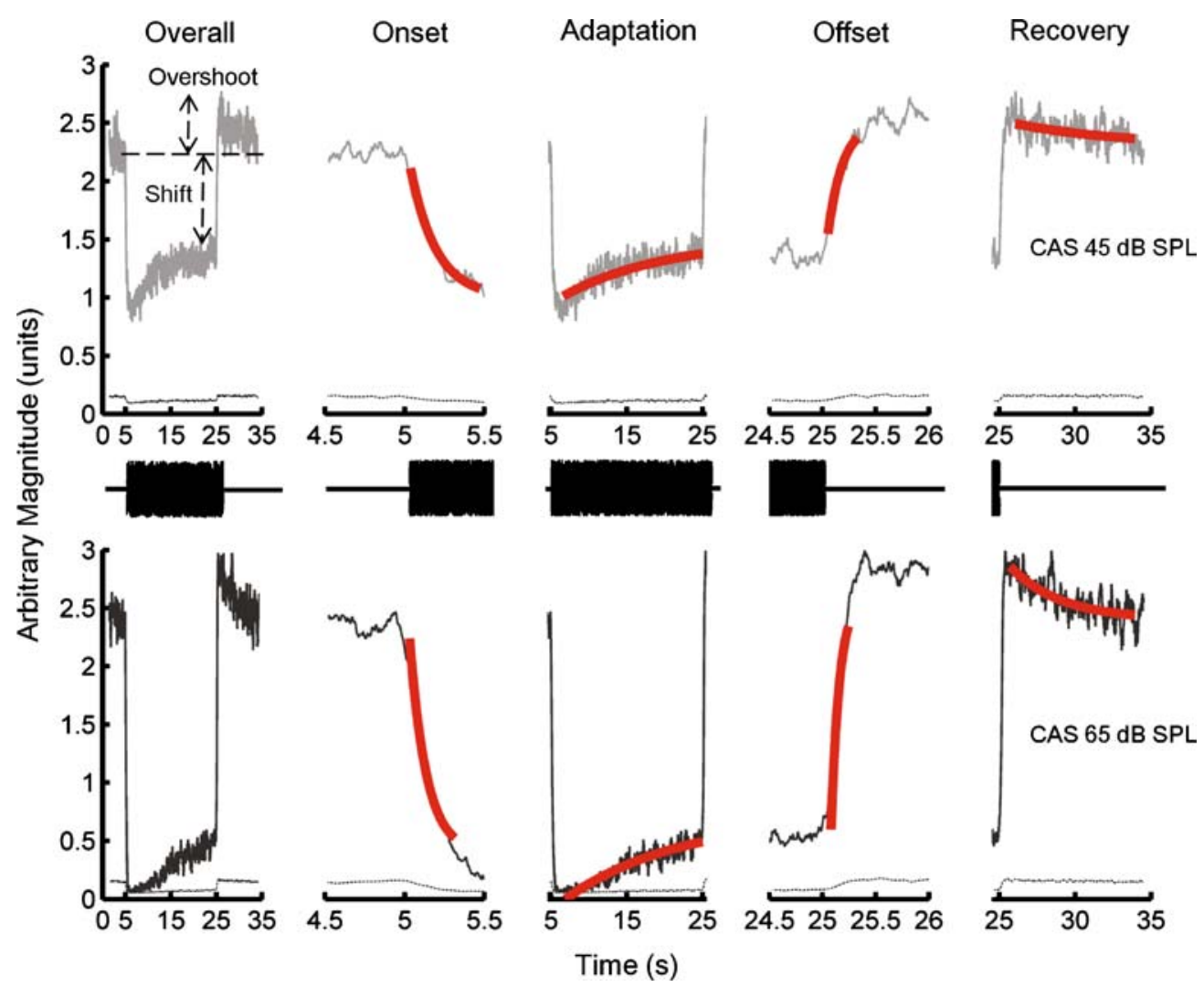

FIG. 6. An example (subject 2) of the time course of SOAE magnitude shifts with CAS at 45 (upper row) or $65 \mathrm{~dB}$ SPL (lower row). The quantification of shift and overshoot in magnitude is illustrated in the upper left panel. Shifts at CAS onset, during the presence of CAS (adaptation), at CAS offset and after CAS offset (recovery) are illustrated in columns two through five. The shifts were

vation in frequency) at CAS onset, a gradual release from elevation (adaptation) in the presence of CAS, a rapid overshoot at CAS offset, and a gradual return to the baseline (recovery). Two examples from subjects 1 and 3 are presented in Figure 7. At baseline, the examined SOAE in subject 1 (Fig. 7, left panel) was $13 \mathrm{~dB}$ SPL at $1,426 \mathrm{~Hz}$. The SOAE examined in subject 3 was $22.4 \mathrm{~dB}$ SPL at 2,026 $\mathrm{Hz}$ (Fig. 7, right panel). In both cases, the change in SOAE frequency was generally bigger with increasing CAS level. However, the frequency elevation appears to saturate for CAS levels greater than $55 \mathrm{~dB}$ SPL in subject 3 (Fig. 7, right panel). The SOAE was reduced to the noise floor immediately after the onset of CAS at $75 \mathrm{~dB}$ SPL in subject 1, thereby preventing estimation of its nominal frequency (marked by arrow in Fig. 7, left panel). As in the case of magnitude changes, SOAE frequency elevation, adaptation, and overshoot varied among subjects. Group data are presented later in the paper.

The MEM reflex threshold was estimated to be $70 \mathrm{~dB}$ SPL for both subjects 1 and 3. Thus, we attribute the changes in SOAE frequency to the MOC reflex for CAS levels up to $65 \mathrm{~dB}$ SPL, and to fitted with a one-exponential function (thick red lines, Eqs. 5 and 6). Note that the SOAE was suppressed into the noise floor after the onset of CAS at $65 \mathrm{~dB}$ SPL, thereby affecting the time constant of the fitted curve. The time constant of adaptation for this condition was therefore not included in group data (Fig. 10).

a combination of MOC and MEM reflexes for CAS at $75 \mathrm{~dB}$ SPL.

Figure 8 illustrates the quantification of the frequency shift and overshoot in subject 3. Fitting of oneexponential curves to various stages of CAS-induced change in SOAE frequency are depicted for subject 3 in the last four columns of Figure 8. The top and bottom rows of Figure 8 represent results for CAS levels of 45 and $65 \mathrm{~dB}$ SPL, respectively. Group data for shift, overshoot, as well as time constants for various stages are presented below.

\section{Group data}

In Figure 9, shifts (left column) and overshoot (right column) in SOAE magnitude (top row) and frequency (bottom row) from all subjects (color coded) as a function of CAS level are presented. In each panel, the data points following a dashed line are likely to be affected by the MEM reflex. For example, the MEM reflex threshold was estimated to be at $50 \mathrm{~dB}$ SPL for subject 4 . Hence, all data points from this subject are connected using dashed lines. In contrast, all other subjects had MEM reflex thresholds 


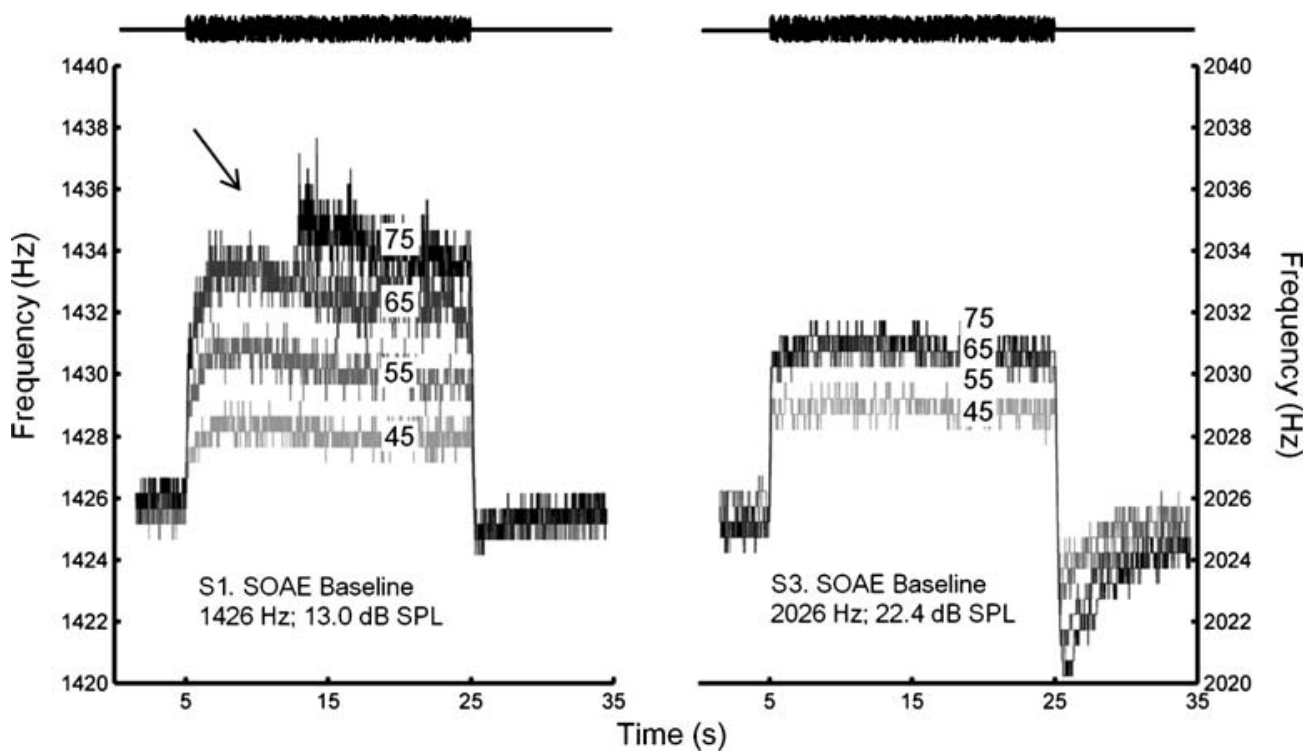

FIG. 7. Two examples of shifts in SOAE frequency as a function of time for different CAS levels. In the left panel, the onset of the contralateral noise at $75 \mathrm{~dB}$ SPL suppressed the SOAE into the noise floor, resulting in a lack of nominal SOAE frequency and a gap in the trace (marked by the arrow).

at $70 \mathrm{~dB}$ SPL. Thus, only the data point obtained with CAS of $75 \mathrm{~dB}$ SPL follows a dashed line.

As CAS level increased, both magnitude shift (reduction) and frequency shift (elevation) increased (Fig. 9, left column). SOAEs from subjects 4 and 5 were fully reduced into the noise floor by all CAS levels, and the noise floor level was arbitrarily taken as the SOAE magnitude during CAS. As a result, their growth curves for magnitude shift did not change as a function of CAS level, and their nominal SOAE frequencies were undeterminable (data not shown in bottom left panel). We chose to visually separate subjects 4 and 5 in the top left panel of Figure 9 by using open symbols. Notice that the magnitude is presented in $\mathrm{dB}$ re: baseline. The arbitrary magnitude in the Choi-Williams distribution represents energy density whose square root is roughly on the same scale as sound pressure. The non-local nature of the ChoiWilliams distribution prevents a strict definition of this relationship. Thus, estimation of $\mathrm{dB}$ change on the SPL scale should be performed on the square root of the output of the Choi-Williams distribution. The overshoot is in the positive direction for magnitude and in the negative direction for frequency, and is generally greater for higher CAS levels (Fig. 9, right column).

By fitting one-exponential curves to SOAE data (Figs. 6 and 8), we also quantified the time constants of SOAE shifts in both magnitude and frequency for CAS onset $\left(\tau_{\text {onset }}\right)$, adaptation $\left(\tau_{\text {adaptation }}\right)$, offset $\left(\tau_{\text {offset }}\right)$, and recovery $\left(\tau_{\text {recovery }}\right)$. Time constants are displayed in Figure 10 as a function of CAS level. The trends in the time-constant growth functions are less clear than those in SOAE shift/overshoot growth functions (Fig. 9). Nevertheless, for both SOAE magnitude and frequency shifts, the onset time constants $\left(\tau_{\text {onset }}\right)$ decrease as the CAS level increases (Fig. 10, left column). Subject 2 showed a frequency shift of less than $1 \mathrm{~Hz}$, thus our frequency resolution did not allow us to fit the one-exponential curve for this subject. SOAEs from subjects 4 and 5 were completely reduced into the noise floor by CAS, leaving the SOAE frequency undeterminable. Therefore, these three subjects were excluded from the time constant estimation for SOAE frequency changes (Fig. 10, lower row), except for recovery data for subject 4. No general patterns were observed for adaptation, offset, and recovery in either magnitude or frequency.

\section{DISCUSSION}

The principal focus of the experiments presented here was to examine the time course of changes in magnitude and frequency of SOAEs in response to CAS, which are arguably mediated through the MOC efferents. We attempted to carefully characterize the influence of the MEM reflex on our results. The use of SOAEs to characterize the effects of the MOC system on human cochlear mechanics may be advantageous as external stimulation, which could activate the MOC, is not required.

In agreement with previous studies, we observed not only a reduction in SOAE magnitude and elevation in SOAE frequency due to CAS, but also an adaptation following the shift at CAS onset for 


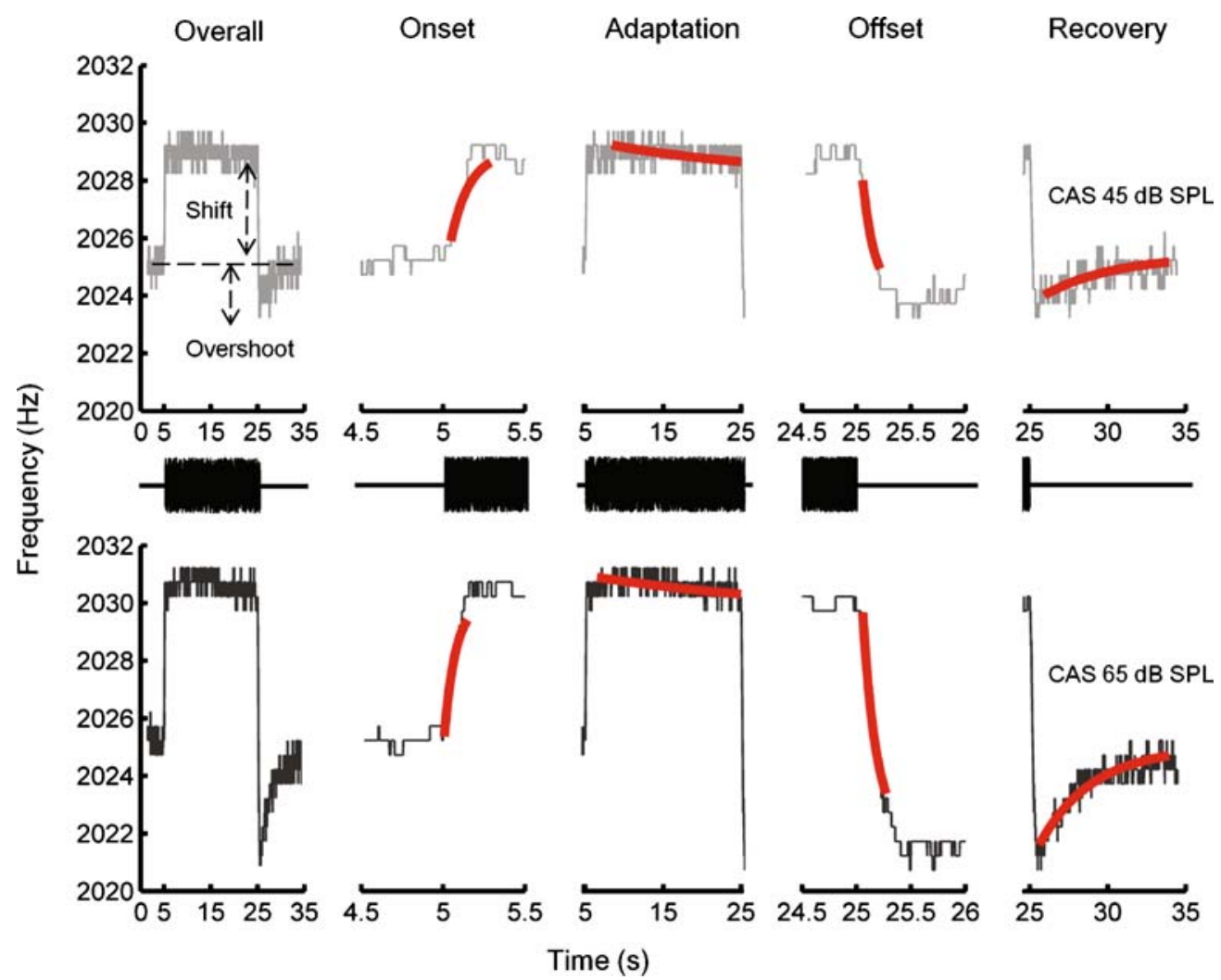

FIG. 8. An example (subject 3) of the time course of SOAE frequency shifts with contralateral noise at 45 (upper row) or $65 \mathrm{~dB}$ SPL (lower row), respectively. The quantification of frequency shift and overshoot is illustrated in the upper left panel. Shifts at CAS

both SOAE magnitude and frequency (Mott et al. 1989; Harrison and Burns 1993). Although some of the general trends observed here were also observed in previous reports, the time course of these events was not fully characterized due to the coarse temporal resolution of the analysis tools employed. Smurzynski et al. (2002) overcame the challenge of acute temporal as well as spectral resolution by using a modified Wigner-Ville distribution, a member of the Cohen's class distribution function, to examine changes in SOAE magnitude and frequency. They reported the onset time constants of fitted oneexponential curves for both magnitude and frequency changes of SOAEs due to broadband CAS at $65 \mathrm{~dB}$ SPL. Note the general agreement of these data with those of the current study in the left column of Figure 10. Here, we extended the findings of the previous work by characterizing the time course of all stages of CASinduced changes in SOAE magnitude and frequency. Particularly, we have identified an overshoot after CAS offset, for both SOAE magnitude and frequency. Furthermore, by examining CAS-induced changes over a range of CAS levels, we have attempted to document the growth pattern of changes in each stage.

The CAS-induced reduction in OAE magnitude is observed in other OAE types, such as DPOAEs onset, during the presence of CAS (adaptation), at CAS offset and after CAS offset (recovery) are illustrated in columns two through five. The shifts were fitted with a one-exponential function (thick red lines, Eqs. 5 and 6).

(Liberman et al. 1996), SFOAEs (Guinan et al. 2003), and TEOAEs (Liberman 1989; Collet et al. 1990). The changes observed in OAEs due to CAS are arguably mediated via the MOC which attenuates the gain of the cochlear amplifier. This reduction in the gain of the cochlear amplifier is directly reflected in the reduction of basilar membrane vibration due to MOC activation (Murugasu and Russell 1996).

The mechanism underlying the CAS-induced elevation in SOAE frequency has not been extensively discussed in the literature. Mott et al. (1989) proposed that the MOC activity increases OHC membrane conductance thereby changing the phase of the feedback force provided by OHCs and altering the intrinsic tuning of the emission generator. It should be noted that the OHCs themselves were not required to be tuned in the Mott et al. (1989) model as they serve merely as the power source of the SOAE generator. In another model of SOAE generation based on cochlear standing-waves, SOAEs are generated by a population of OHCs, and gain sufficient magnitude to escape the cochlea due to appropriate phase accumulation following multiple reflections between the region of generation and the cochlear boundary with the middle ear (Shera 2003). Tuning of the emission generator thus depends on the round- 


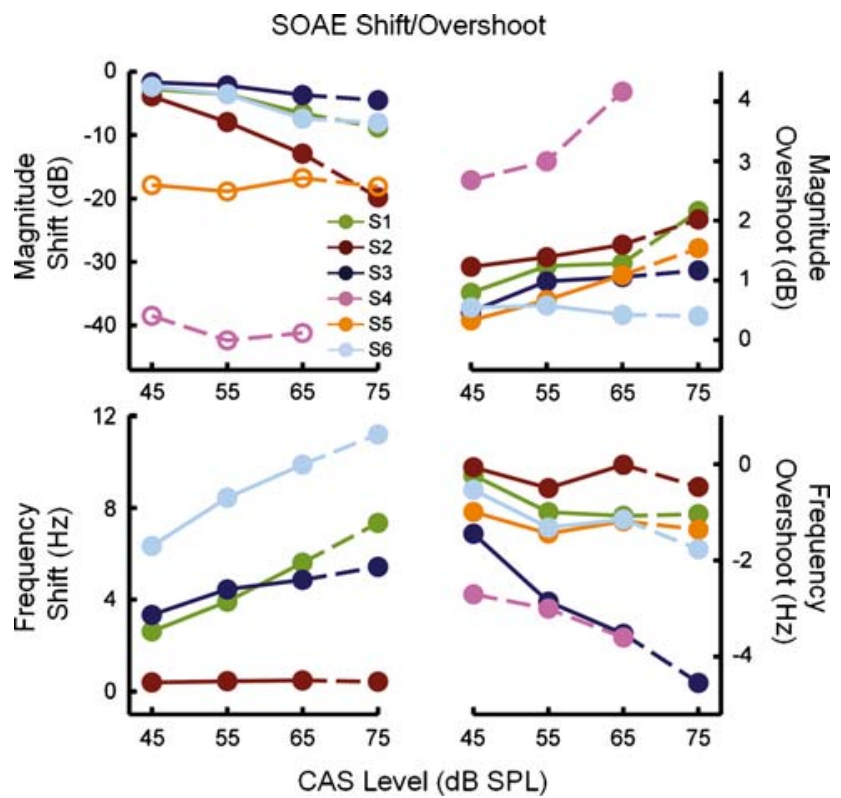

FIG. 9. CAS-induced shift (left column) and overshoot (right column) in both magnitude (upper row) and frequency (lower row) as a function of CAS level. The shift in magnitude was converted to $\mathrm{dB}$ re: baseline. Dashed lines indicate potential contamination from the MEM reflex given the experimental MEM reflex thresholds (see text and Table 1). Overall, as CAS level increases, shifts and overshoots in both magnitude and frequency increase. SOAEs from subject 4 and 5 were completely suppressed into the noise floor by all levels of CAS. Hence, their magnitude shifts were invariant as a function of CAS level (open symbols) and their data are excluded from the bottom left panel.

trip phase accumulation of the cochlear traveling wave. The so-called fast effect of MOC activity has been shown to cause a phase lead in basilar membrane vibration (Murugasu and Russell 1996; Cooper and Guinan 2003). Such a phase change would move the 'in-phase' coherent reflection region in Shera's cochlear "resonant cavity" model towards the basal end, hence increasing SOAE frequencies.

\section{Adaptation during the presence of noise}

We observed a partial release (adaptation) from the initial change in SOAE magnitude and frequency after CAS onset. Such adaptation has been reported previously (Mott et al. 1989; Harrison and Burns 1993), but its exact time course has not been documented due to limitations of analysis techniques. Similar adaptation patterns due to MOC activation, while on different time scales, have been reported in both compound action potential (CAP) and basilar membrane vibration recordings in experimental animals (Sridhar et al. 1995; Cooper and Guinan 2003).

The term adaptation has been used in connection with DPOAEs and the MOC in a different connotation. The magnitude of DPOAEs gradually reduces after stimulus onset even in the absence of CAS
(Liberman et al. 1996; Kim et al. 2001; Kujawa and Liberman 2001; Relkin et al. 2005). In contrast, the term adaptation is used in this paper to signify a release from MOC-induced changes in SOAEs.

Decay of the MEM reflex could be suspected as the cause of the SOAE adaptation observed during CAS (Mott et al. 1989). However, in agreement with Mott et al. (1989), we discount the role of MEM reflex decay because adaptation was observed well below our best estimates of the MEM reflex threshold.

Another postulation is that the observed SOAE adaptation is synaptic in origin. As is well known, the main neurotransmitter of the MOC pathway is acetylcholine (Eybalin 1993). The receptor of acetylcholine in the auditory efferent system is a special nicotinic acetylcholine receptor composed of $\alpha 9$ and a10 subunits (Elgoyhen et al. 1994; Elgoyhen et al. 2001). Continuous activation of the MOC efferent may lead to depression of neurotransmitter release due to a depletion of presynaptic vesicles that are ready to be released, thereby causing a reduction in postsynaptic current amplitude and weakened synaptic strength (Zucker and Regehr 2002).

Yet another contributor could be the desensitization of the postsynaptic nicotinic acetylcholine receptors (Elgoyhen et al. 1994; Elgoyhen et al. 2001). Elgoyhen et al. (2001) expressed $\alpha 9 / \alpha 10$ receptors in Xenopus oocytes and found an immediate decrease of the recorded current in the presence of continuous extracellular acetylcholine.

A similar pattern of adaptation is also observed in experiments where SOAEs are suppressed by an ipsilateral external tone (Murphy et al. 1995). But these changes occur at a much faster time scale and are arguably due to the mechanical interplay between the target SOAE, the external tone, and another SOAE in frequency proximity.

We fitted one-exponential curves to the adaptation traces (see Figs. 6, 8, and 10). However, no consistent trends in the time constant of adaptation were observed as a function of CAS level.

\section{Overshoot at the offset of contralateral stimulation}

We have reported an overshoot for both SOAE magnitude and frequency at the offset of contralateral noise. The overshoot in SOAE magnitude reported in Murphy et al. (1995) at the offset of an external tone in the ipsilateral ear was explained by the suppression from a neighboring higher-frequency SOAE which responds more slowly to the external tone. This model of differential responses of two neighboring SOAEs cannot explain our results where changes in SOAE were evoked by contralateral stimulation. We observed prominent overshoot in both SOAE magnitude and frequency in subjects where no SOAEs of 


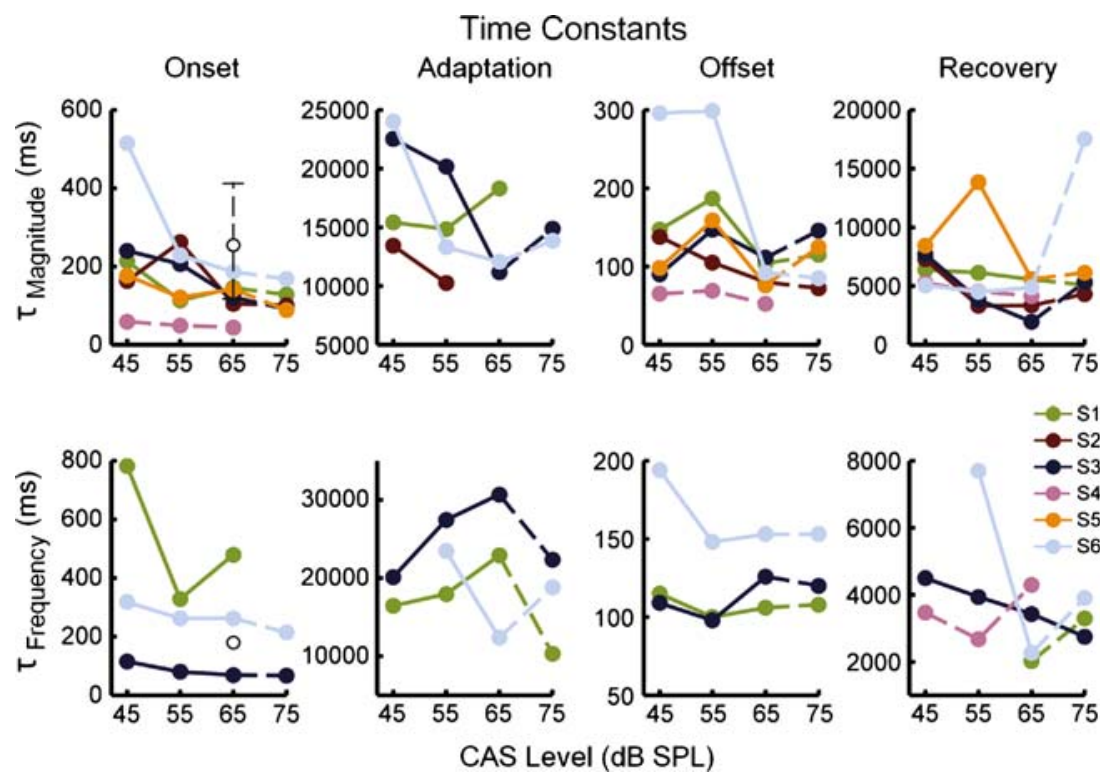

FIG. 10. Time constant $\tau$ of the fitted curves of SOAE shifts as a function of CAS level. Onset, adaptation, offset, and recovery time constants are presented in columns one through four. Time constants of shifts in magnitude and frequency are presented in the upper and lower rows, respectively. Dashed lines indicate potential contamination from the MEM reflex given the experimental MEM reflex thresholds (Table 1). Subject 2 demonstrated a frequency shift of less than $1 \mathrm{~Hz}$. The SOAEs from subjects 4 and 5 were suppressed into

any significance were measurable near the target SOAE. We postulate that other mechanisms account for the SOAE overshoot observed in this study.

Following exposure to a loud ipsilateral low-frequency tone, a bounce in the recovery of hearing thresholds, as well as a concomitant bounce in otoacoustic emissions and cochlear microphonics, has been reported previously in the literature (Hirsh and Ward 1952; Kemp 1986; Kirk et al. 1997; Kirk and Patuzzi 1997; Kevanishvili et al. 2006). This bounce, on a time scale of minutes, has been identified in different types of OAEs, including SOAEs, TEOAEs, and DPOAEs. Kirk et al. (1997) suggested that the shift of the operating point on the OHC transfer function, rather than neural activity, is responsible for this bounce. However, it is uncertain whether this mechanism accounts for the bounce phenomenon reported here, which is on a much faster time scale of seconds.

Similar overshoots have been observed in SFOAEs, TEOAEs as well as the slow MOC effect on CAP and sound-evoked basilar membrane vibration, following either CAS or electrical stimulation of the MOC efferents (Sridhar et al. 1995; Cooper and Guinan 2003; Backus and Guinan 2006; Zyl et al. 2009). The overshoot in CAP, sound-evoked basilar membrane vibration and TEOAEs, which builds up and decays over hundreds of seconds, is significantly different the noise floor for all CAS levels. Thus, the time constants of the frequency shift from these three subjects are not available except for the recovery stage. Overall, at CAS onset there is a negative slope as a function of CAS level. The trends in other panels are less prominent, especially for adaptation and recovery due to their low fitting coefficients. Onset time constants obtained from the WignerVille distribution by Smurzynski et al. (2002) are marked by black open circles (median $\tau$ ) and horizontal bars (range of $\tau$ ).

from the overshoot observed in SOAEs and SFOAEs, which builds up and decays in a few seconds.

\section{Time constants compared with previous studies}

We fitted various stages of the CAS-induced changes in SOAE with one-exponential functions and found great variation across subjects and CAS levels $\left(44 \mathrm{~ms}<\tau_{\text {onset }}<782 \mathrm{~ms}, 10.3 \mathrm{~s}<\tau_{\text {adaptation }}<30.7 \mathrm{~s}\right.$, $52 \mathrm{~ms}<\tau_{\text {offset }}<299 \mathrm{~ms}, 1.9 \mathrm{~s}<\tau_{\text {recovery }}<17.5 \mathrm{~s}$ ). These values of $\tau_{\text {onset }}$ are comparable to those reported by Smurzynski et al. (2002). Unfortunately, they did not document the time constants of other stages of SOAE shift. Nonetheless, comparison of our data with changes in evoked OAEs is possible. The time course of DPOAE onset adaptation (without CAS) fitted with a two-exponential function has been reported in the literature (Kim et al. 2001; Bassim et al. 2003). The median values of the fast and slow time constants were $69 \mathrm{~ms}$ and $1.51 \mathrm{~s}$ in Kim et al. (2001), and $72.9 \mathrm{~ms}$ and $2.1 \mathrm{~s}$ in Bassim et al. (2003). The onset and offset time constants of DPOAE suppression by contralateral noise were similar to those of DPOAE onset adaptation (Bassim et al. 2003). These fast time constants are comparable to the faster subset of $\tau_{\text {onset }}$ and $\tau_{\text {offset }}$ values reported here for SOAEs. Unlike our results, no DPOAE adaptation (gradual increase in amplitude) during CAS or overshoot after CAS offset was 
observed in either the Kim et al. (2001) or the Bassim et al. (2003) report. Backus and Guinan (2006) have reported onset and offset time constants of $277 \pm$ $62 \mathrm{~ms}$ and $159 \pm 54 \mathrm{~ms}$, respectively, for changes in SFOAE level with CAS. These values overlap with the slower subset of onset and offset time constants reported here for SOAEs. Additionally, Backus and Guinan (2006) identified an SFOAE overshoot following CAS but not adaptation during CAS.

\section{Influence of MEM reflex}

The possible influence of the MEM reflex in experiments investigating MOC functions or OAEs has been a source of concern. Using an alternate approach we were able to detect the MEM reflex at lower stimulation levels than those detectable using a commercial impedance audiometer. Subject 4 had an MEM reflex threshold at $50 \mathrm{~dB}$ SPL and data from this subject are clearly separated from the remaining data pool (Fig. 9, top row). The remaining subjects had MEM reflex thresholds around $70 \mathrm{~dB}$ SPL, just below our highest CAS level. We did not observe any dramatic change of slope in the growth functions of magnitude and frequency shift or overshoot (Fig. 9) as CAS increased beyond the supposed MEM reflex threshold in these subjects. Middle-ear muscle activation has been associated with a dramatic change of slope in some studies (Guinan et al. 2003) but not others (Sun 2008a). The lack of dramatic slope change in our results could mean that all effects shown here are dominated by either the MEM reflex or the MOC reflex, or that these two effects are qualitatively continuous.

The MEM reflex threshold measured with our method ranged from 50 to $70 \mathrm{~dB}$ SPL. These results were consistently lower than those obtained with the commercial middle-ear analyzer (Interacoustics Audio Traveller AA220). Two previous papers have developed SFOAE-based techniques to measure the MEM reflex threshold (Guinan et al. 2003; Goodman and Keefe 2006). In both of these publications, the lowest elicitor level shown to evoke the MEM reflex was between 55 and $60 \mathrm{~dB}$ SPL. A comparison between laboratory methods is perhaps premature given the small sample sizes of these reports, including ours. However, these laboratory methods are unequivocally more sensitive to the MEM reflex than a clinical middle-ear analyzer.

\section{ACKNOWLEDGMENTS}

The authors would like to thank Rebekah Abel, Rachael Baiduc, Renee Banakis, and Abby Rogers for their constructive comments on a previous version of this paper.
Helpful discussions with Jonathan Siegel and Mario Ruggero shaped our thinking about the issues presented here. This research was partially funded by NIDCD R01 DC008420. Parts of this work were presented at the 2009 annual convention of the American Auditory Society.

\section{REFERENCES}

Backus BC, Guinan JJ JR (2006) Time-course of the human medial olivocochlear reflex. J. Acoust. Soc. Am. 119:2889-2904

Bassim MK, Miller RL, Buss E, Sмith DW (2003) Rapid adaptation of the 2f1-f2 DPOAE in humans: binaural and contralateral stimulation effects. Hear Res 182:140-152

Brown MC (1989) Morphology and response properties of single olivocochlear fibers in the guinea pig. Hear. Res. 40:93-109

Burns EM, Harrison WA, Bulen JC, Keefe DH (1993) Voluntary contraction of middle ear muscles: effects on input impedance, energy reflectance and spontaneous otoacoustic emissions. Hear. Res. 67:117-127

CoHen L (1995) Time-frequency analysis. Prentice Hall, Englewood Cliffs

Collet L, Kemp DT, Veuillet E, Duclaux R, Moulin A, Morgon A (1990) Effect of contralateral auditory stimuli on active cochlear micro-mechanical properties in human subjects. Hear. Res. 43:251-261

CoOper NP, Guinan JJ JR (2003) Separate mechanical processes underlie fast and slow effects of medial olivocochlear efferent activity. J. Physiol. 548:307-312

Dallos P (1992) The active cochlea. J. Neurosci. 12:4575-4585

Dolan DF, Guo MH, Nuttall AL (1997) Frequency-dependent enhancement of basilar membrane velocity during olivocochlear bundle stimulation. J. Acoust. Soc. Am. 102:3587-3596

Elgoyhen AB, Johnson DS, Boulter J, Vetter DE, Heinemann S (1994) Alpha 9: an acetylcholine receptor with novel pharmacological properties expressed in rat cochlear hair cells. Cell 79:705-715

Elgoyhen AB, Vetter DE, Katz E, Rothlin CV, Heinemann SF, Boulter J (2001) Alpha10: a determinant of nicotinic cholinergic receptor function in mammalian vestibular and cochlear mechanosensory hair cells. Proc. Natl. Acad. Sci. USA 98:3501-3506

Erostegui C, Norris CH, BobBin RP (1994) In vitro pharmacologic characterization of a cholinergic receptor on outer hair cells. Hear. Res. 74:135-147

Evans MG (1996) Acetylcholine activates two currents in guinea-pig outer hair cells. J. Physiol. 491 (Pt 2):563-578

EybaLIN M (1993) Neurotransmitters and neuromodulators of the mammalian cochlea. Physiol. Rev. 73:309-373

Feeney MP, KeEFe DH, Marryott LP (2003) Contralateral acoustic reflex thresholds for tonal activators using wideband energy reflectance and admittance. J. Speech. Lang. Hear. Res. 46:128136

Fex J (1962) Auditory activity in centrifugal and centripetal cochlear fibres in cat. A study of a feedback system. Acta. Physiol. Scand. Suppl. 189:1-68

Fuchs PA, Murrow BW (1992) Cholinergic inhibition of short (outer) hair cells of the chick's cochlea. J. Neurosci. 12:800-809

Galambos R (1956) Suppression of auditory nerve activity by stimulation of efferent fibers to cochlea. J. Neurophysiol. 19:424-437

Garinis AC, Glattke T, Cone-Wesson BK (2008) TEOAE suppression in adults with learning disabilities. Int. J. Audiol. 47:607-614

GELfand SA (1984) The contralateral acoustic reflex. In: Silman S (ed) The acoustic reflex: basic principles and clinical applications. Academic, Orlando, pp 137-186 
GifFord ML, Guinan JJ JR (1987) Effects of electrical stimulation of medial olivocochlear neurons on ipsilateral and contralateral cochlear responses. Hear. Res. 29:179-194

Giraud AL, Collet L, Cherr-Croze S, Magnan J, Chays A (1995) Evidence of a medial olivocochlear involvement in contralateral suppression of otoacoustic emissions in humans. Brain. Res. 705:15-23

Goodman SS, KeEFe DH (2006) Simultaneous measurement of noiseactivated middle-ear muscle reflex and stimulus frequency otoacoustic emissions. J. Assoc. Res. Otolaryngol. 7:125-139

Guinan JJ JR (2006) Olivocochlear efferents: anatomy, physiology, function, and the measurement of efferent effects in humans. Ear. Hear. 27:589-607

Guinan JJ Jr, Backus BC, Lilaonitkul W, Aharonson V (2003) Medial olivocochlear efferent reflex in humans: otoacoustic emission (OAE) measurement issues and the advantages of stimulus frequency OAEs. J. Assoc. Res. Otolaryngol. 4:521-540

HARrison WA, Burns EM (1993) Effects of contralateral acoustic stimulation on spontaneous otoacoustic emissions. J. Acoust. Soc. Am. 94:2649-2658

HIRSH IJ, WARD WD (1952) Recovery of the auditory threshold after strong acoustic stimulation. J. Acoust. Soc. Am. 24:131-141

Hood LJ, Berlin CI, Bordelon J, Rose K (2003) Patients with auditory neuropathy/dys-synchrony lack efferent suppression of transient evoked otoacoustic emissions. J. Am. Acad. Audiol. 14:302-313

Housley GD, Ashmore JF (1991) Direct measurement of the action of acetylcholine on isolated outer hair cells of the guinea pig cochlea. Proc. Biol. Sci. 244:161-167

Kakehata S, Nakagawa T, Takasaka T, Akaike N (1993) Cellular mechanism of acetylcholine-induced response in dissociated outer hair cells of guinea-pig cochlea. J. Physiol. 463:227-244

KemP DT (1986) Otoacoustic emissions, travelling waves and cochlear mechanisms. Hear. Res. 22:95-104

Kevanishvili Z, Hofmann G, Burdzgla I, Pietsch M, Gamgebeli Z, Yarin Y, TushishVIli M, ZahNert T (2006) BehaVior of EVOKED OTOACOUSTIC EMISSION UNDER LOW-FREQUENCY TONE EXPOSURE: OBJECTIVE STUDY OF THE BOUNCE PHENOMENON IN HUMANS. Hear. Res. 222:62-69

Kim DO, Dorn PA, Neely ST, Gorga MP (2001) Adaptation of distortion product otoacoustic emission in humans. J. Assoc. Res. Otolaryngol. 2:31-40

Kirk DL, PATUZZI RB (1997) Transient changes in cochlear potentials and DPOAEs after low-frequency tones: the 'two-minute bounce' revisited. Hear. Res. 112:49-68

Kirk DL, Moleirinho A, PAtuZzi RB (1997) Microphonic and DPOAE measurements suggest a micromechanical mechanism for the 'bounce' phenomenon following low-frequency tones. Hear. Res. 112:69-86

Kujawa SG, Liberman MC (2001) Effects of olivocochlear feedback on distortion product otoacoustic emissions in guinea pig. J. Assoc. Res. Otolaryngol. 2:268-278

LiBERMAN MC (1989) Rapid assessment of sound-evoked olivocochlear feedback: suppression of compound action potentials by contralateral sound. Hear. Res. 38:47-56

Liberman MC, Brown MC (1986) Physiology and anatomy of single olivocochlear neurons in the cat. Hear. Res. 24:17-36

Liberman MC, Puria S, Guinan JJ Jr (1996) The ipsilaterally evoked olivocochlear reflex causes rapid adaptation of the 2f1-f2 distortion product otoacoustic emission. J. Acoust. Soc. Am. 99:3572-3584

Lilaonitkul W, Guinan JJ Jr (2009) Human Medial Olivocochlear Reflex: Effects as Functions of Contralateral, Ipsilateral, and Bilateral Elicitor Bandwidths. J Assoc Res Otolaryngol 10:459470
Long GR, Talmadge CL (1997) Spontaneous otoacoustic emission frequency is modulated by heartbeat. J. Acoust. Soc. Am. 102:2831-2848

Maison S, Micheyl C, Andeol G, Gallego S, Collet L (2000) Activation of medial olivocochlear efferent system in humans: influence of stimulus bandwidth. Hear. Res. 140:111-125

Maison SF, Adams JC, Liberman MC (2003) Olivocochlear innervation in the mouse: immunocytochemical maps, crossed versus uncrossed contributions, and transmitter colocalization. J. Comp. Neurol. 455:406-416

Mott JB, Norton SJ, Neely ST, Warr WB (1989) Changes in spontaneous otoacoustic emissions produced by acoustic stimulation of the contralateral ear. Hear. Res. 38:229-242

Moulin A, Collet L, Duclaux R (1993A) Contralateral auditory stimulation alters acoustic distortion products in humans. Hear Res 65:193-210

Moulin A, Collet L, Duclaux R (1993B) Contralateral auditory stimulation alters acoustic distortion products in humans. Hear. Res. 65:193-210

Mountain DC (1980) Changes in endolymphatic potential and crossed olivocochlear bundle stimulation alter cochlear mechanics. Science 210:71-72

Muller-Wehlau M, Mauermann M, Dau T, Kollmeier B (2005) The effects of neural synchronization and peripheral compression on the acoustic-reflex threshold. J. Acoust. Soc. Am. 117:3016-3027

Murphy WJ, Tubis A, Talmadge CL, Long GR (1995) Relaxation dynamics of spontaneous otoacoustic emissions perturbed by external tones. II. Suppression of interacting emissions. J. Acoust. Soc. Am. 97:3711-3720

Murugasu E, Russell IJ (1996) The effect of efferent stimulation on basilar membrane displacement in the basal turn of the guinea pig cochlea. J. Neurosci. 16:325-332

Neumann J, Uppenkamp S, Kollmeier B (1996) Detection of the acoustic reflex below $80 \mathrm{~dB}$ HL. Audiol. Neurootol. 1:359-369

Norman M, Thornton AR (1993) Frequency analysis of the contralateral suppression of evoked otoacoustic emissions by narrow-band noise. Br. J. Audiol. 27:281-289

Oliver D, Klocker N, Schuck J, Baukrowitz T, Ruppersberg JP, Fakler B (2000) Gating of $\mathrm{Ca}^{2+}$-activated $\mathrm{K}^{+}$channels controls fast inhibitory synaptic transmission at auditory outer hair cells. Neuron 26:595-601

Relkin EM, Sterns A, Azeredo W, Prieve BA, Woods CI (2005) Physiological mechanisms of onset adaptation and contralateral suppression of DPOAEs in the rat. J. Assoc. Res. Otolaryngol. 6:119-135

Robertson D, Gummer M (1985) Physiological and morphological characterization of efferent neurones in the guinea pig cochlea. Hear. Res. 20:63-77

Robles L, Delano PH (2008) Efferent system. In: Dallos P, Oertel D (eds) The senses: a comprehensive reference. Academic, New York

SHERA CA (2003) Mammalian spontaneous otoacoustic emissions are amplitude-stabilized cochlear standing waves. J. Acoust. Soc. Am. 114:244-262

Siegel JH, Kim DO (1982) Efferent neural control of cochlear mechanics? Olivocochlear bundle stimulation affects cochlear biomechanical nonlinearity. Hear. Res. 6:171-182

Smurzynski J, Lisowska G, Grzanka A, Namysloski G 2002. Dynamic changes in spontaneous otoacoustic emissions produced by contralateral broadband noise. Biophysics of the Cochlea, Proc Int Symp, Titisee, Germany

Sridhar TS, Liberman MC, Brown MC, Sewell WF (1995) A novel cholinergic "slow effect" of efferent stimulation on cochlear potentials in the guinea pig. J. Neurosci. 15:3667-3678

Sun XM (2008A) Contralateral suppression of distortion product otoacoustic emissions and the middle-ear muscle reflex in human ears. Hear. Res. 237:66-75 
Sun XM (2008B) Distortion product otoacoustic emission fine structure is responsible for variability of distortion product otoacoustic emission contralateral suppression. J. Acoust. Soc. Am. 123:4310-4320

Velenovsky DS, GLatTKe TJ (2002) The effect of noise bandwidth on the contralateral suppression of transient evoked otoacoustic emissions. Hear. Res. 164:39-48

Veuillet E, Collet L, Duclaux R (1991) Effect of contralateral acoustic stimulation on active cochlear micromechanical properties in human subjects: dependence on stimulus variables. J. Neurophysiol. 65:724-735

Whitehead ML, Martin GK, Lonsburi-Martin BL (1991) Effects of the crossed acoustic reflex on distortion-product otoacoustic emissions in awake rabbits. Hear. Res. 51:55-72
Wiederhold ML, Kiang NY (1970) Effects of electric stimulation of the crossed olivocochlear bundle on single auditory-nerve fibers in the cat. J. Acoust. Soc. Am. 48:950-965

Williams DM, Brown AM (1997) The effect of contralateral broadband noise on acoustic distortion products from the human ear. Hear. Res. 104:127-146

Yoshida N, Liberman MC, Brown MC, Ssewell WF (2001) Fast, but not slow, effects of olivocochlear activation are resistant to apamin. J. Neurophysiol. 85:84-88

Zucker RS, Regehr WG (2002) Short-term synaptic plasticity. Annu. Rev. Physiol. 64:355-405

ZyL AV, S wanepoel DW, Hall JW 3RD (2009) Effect of prolonged contralateral acoustic stimulation on transient evoked otoacoustic emissions. Hear. Res. 254:77-81 